\title{
Prevalence of thyroid dysfunction in patients with Type 2 diabetes mellitus and its correlation with insulin resistance and serum markers for autoimmune thyroiditis
}

\author{
Sulagna Sahu ${ }^{1}$, Soumya Kanti Dutta ${ }^{2}$, Shib Shankar Kuiri ${ }^{3}$, Mintu Mohan Nandi ${ }^{4}$, \\ Pranay Kabiraj ${ }^{5}$, Utpal $\mathrm{De}^{6}$ \\ ${ }^{1}$ Senior Resident, Medicine, ${ }^{2}$ RMO Cum Clinical Tutor, Medicine, ${ }^{3}$ RMO Cum Clinical Tutor, General Surgery, ${ }^{4}$ Assistant Professor, General \\ Surgery, ${ }^{5}$ Post Graduate Trainee, General Surgery, ${ }^{6}$ Professor and Head, Department General Surgery, B.S. Medical College, Bankura \\ (West Bengal), India
}

\section{A B S T R A C T}

Background: The term "THYROID DIABETES" was coined in early literature to depict the influence of thyroid hormone excess in deterioration of glucose control. Although autoimmune thyroid disease is more prevalent in Type1 Diabetes mellitus as a result of their common origin, the prevalence of hypothyroidism and hyperthyroidism is supposed to be similar to that of general population in patient with type 2 DM. The purpose of the study was to evaluate the prevalence of thyroid dysfunction in patients with type 2 diabetes mellitus and to correlate thyroid abnormalities with insulin resistance and serum markers for autoimmune thyroiditis. Methods: 120 cases of type 2 diabetes mellitus patients satisfying WHO criteria without pre-existing thyroid disease were included in the study. Thyroid function test, fasting serum insulin was done.HOMA-IR \& HOMA-B (HOMA-Homeostatic model assessment) was calculated. Serum antithyroid peroxidase anibody (anti-TPO) and antithyroglobulin antibodies (anti-TG) and ANA were done. Results: Prevalence of thyroid dysfunction in type 2 diabetes mellitus was $28.33 \%$ according to our study, which included overt hypothyroidism (15\%), subclinical hypothyroidism $(8.33 \%)$, secondary hypothyroidism $(0.83 \%)$, overt hyperthyroidism $(1.67 \%)$ and subclinical hyperthyroidism (2.5\%). Anti TPO and anti TG antibodies were elevated in $62.07 \%$ cases of hypothyroidism, $40 \%$ cases of hyperthyroidism and $6.9 \%$ euthyroid cases of type2 DM. Anti TPO and antiTG antibodies were significantly raised in type2DM patient with hypothroidism than that of euthyroid ( $p$ value $<0.0001$ ). Compared to euthyroid diabetics, hypothyroid cases had lower values of insulin resistance markers like fasting insulin, HOMA-IR and HOMA-B. Hyperthyoid cases had higher values. Conclusion: Hyperthyroid diabetics have higher insulin resistance as fasting insulin, HOMA-IR, HOMA-B showed negative correlation with TSH. (p value $<0.05$ )

Key Words: Thyroid diabetes, autoimmune thyroid disease, anti thyroid peroxidase antibody, Homeostatic model assessment for insulin resistance.

\section{INTRODUCTION}

The term "THYROID DIABETES" was coined in early literature to depict the influence of thyroid hormone excess in deterioration of glucose control. Although
Access this article online

Website:

http://nepjol.info/index.php/AJMS

DOI: 10.3126/ajms.v6i6.12603 
with hyperthyroidism. Hypothyroidism is characterized by insulin resistance. ${ }^{1}$ In type $2 \mathrm{DM}$ patients the presence of highly frequent subclinical forms of hyperthyroidism and hypothyroidism should be ruled out since they may be associated with increased risk of nephropathy, retinopathy and cardiovascular events. ${ }^{2}$ The prevalence of subclinical hypothyroidism is higher in patients with metabolic syndrome which can be explained by concomitance of deranged serum lipid concentration, obesity, hypertension, insulin resistance in both these conditions. ${ }^{3}$

Prevalence of Diabetes mellitus in South-East Asia is 8.3\% according to international Diabetes federation. 75 million people have Diabetes.In other words 1 in 12 adults are suffering from Diabetes mellitus. Total Diabetes related deaths are estimated to be 1185.2 thousands in $2014 .^{4}$

According to the Whickham survey, thyroid dysfunction affected $6.6 \%$ of adults (Tunbridge, et al. 1997). ${ }^{5}$ The reported prevalence of thyroid dysfunction in diabetes varied from 2.2 to $17 \%$ in these different works. However, little is known about thyroid size and structure in type 2 diabetes mellitus (Junik et al. 2006). ${ }^{6}$ Although several studies have shown the association between thyroid autoimmunity and type-1 diabetes (Park, et al. 2000; Menon, et al. 2001 $)^{7,8}$ little is known of the risk of thyroid autoimmunity in subjects with type-2 diabetes (MatejkovaBehanova, et al. 2002). ' Studies of Perros et al showed a prevalence of $13.4 \%$ and that of Papazafiropoulou $12.3 \% .{ }^{10} \mathrm{~A}$ recent study in Tumkur, Karnataka showed the prevalence of hypothyroidism in Diabetes mellitus was $12.06 \% .^{11}$

Thyroid hormone act differentially in liver, skeletal muscle and adipose tissue-the main targets of insulin action. While Thyroid hormones oppose the action of insulin and stimulate hepatic gluconeogenesis and glycogenolysis ${ }^{12,13}$ they upregulate the expression of genes such as GLUT-4 and phosphoglycerate kinase involved in glucose transport and glycolysis respectively, thus acting synergistically with insulin facilititating glucose disposal and peripheral utilisation. ${ }^{14,15}$

In hypothyroidism, glucose homeostasis is also affected although its clinical impact is less obvious. Decreased glucose disposal (as compared with euthyroid subjects) has been proved in hypothyroid patients by different methods including clamp studies. ${ }^{16,17}$ Hypothyroidism results in unimpaired ${ }^{18}$ or decreased ${ }^{19,20}$ liver glucose output thereby compensating for insulin resistance present in peripheral tissues and accounting for the diminished insulin requirement for glycaemic control in hypothyroid diabetic patients.
Aims and objectives

1. To Study the prevalence of thyroid dysfunction in patients with type 2 Diabetes mellitus.

2. Correlation of thyroid abnormalities with Insulin resistance and serum markers for autoimmune thyroiditis.

\section{MATERIALS AND METHODS}

\section{Case Selection}

One hundred twenty consecutive cases of type 2 diabetes mellitus patients satisfying WHO (World Health Organisation) criteria without pre-existing thyroid disease attending Medicine Outpatient Department or admitted in department of medicine between September 2012 to September 2013 were taken as cases. Detailed history taking and clinical examination were done in all cases. Thyroid function test including T3,T4,TSH was done by RIA method (IMMUNOTECH).

Fasting serum insulin was done.HOMA-IR \& HOMA-B was calculated as:

HOMA-IR $=[$ FPG $(\mathrm{mg} / \mathrm{dl}) \times$ Fasting Insulin $(\mu \mathrm{U} / \mathrm{ml})] / 405$

HOMA-B $=[360 \times$ Fasting Insulin $(\mu \mathrm{U} / \mathrm{ml})] /[\mathrm{FPG}(\mathrm{mg} / \mathrm{dl})-63]$

Serum autoimmune markers like ANA, anti thyroid peroxidase anibody (anti-TPO) and antithyroglobulin antibodies (anti-TG) were done.

\section{RESULTS}

According to our study, prevalence of thyroid dysfunction in patients with type $2 \mathrm{DM}$ was $28.33 \%$ (34 out of 120 cases) as shown in (Figure 1). Among these 34 cases, 29(24.17\%) were hypothyroid and $5(4.16 \%)$ were hyperthyroid. 86 out of 120 cases $(71.67 \%)$ were euthyroid.

Out of 120 cases of Type 2 diabetes mellitus, 66 were males $(55 \%)$ and 54 were females (45\%).Out of 29 hypothyroid diabetic cases 19 were females and 10 were males as shown in (Figure 2). Out of 5 cases of hyperthyroidism, 3 were females and 2 were males.

Out of 29 cases of hypothyroid diabetics, overt hypothyroidism was found in $18(15 \%)$ patients, subclinical hypothyroidism in $10(8.33 \%)$ cases and secondary hypothyroidism in $1(0.83 \%)$ cases. Among 5 cases of hyperthyroidism, 2(1.67\%) were overt and $3(2.5 \%)$ were subclinical as shown in (Figure 3 ). Twenty-three cases $(19.17 \%)$ were found to have sick euthyroid syndrome.63(52.5\%) patients were euthyroid. So overt hypothyroidism was the major thyroid abnormality in 
type2 DM followed by subclinical hypothyroidism according to our study.

Anti-TPO antibodies were found to be positive in $18(62.07 \%$ ) out of 29 cases of type2 DM with hypothyroidism, 2(40\%) out of 5 cases of hyperthyroid and $6(6.97 \%)$ out of 86 cases of euthyroidism as shown in (Figure 4). These euthyroid diabetic patients with anti TPO antibodies are at risk of developing autoimmune thyroiditis in future. Autoimmune thyroid disease is characterized by lymphocytic infiltration.TPO antibodies

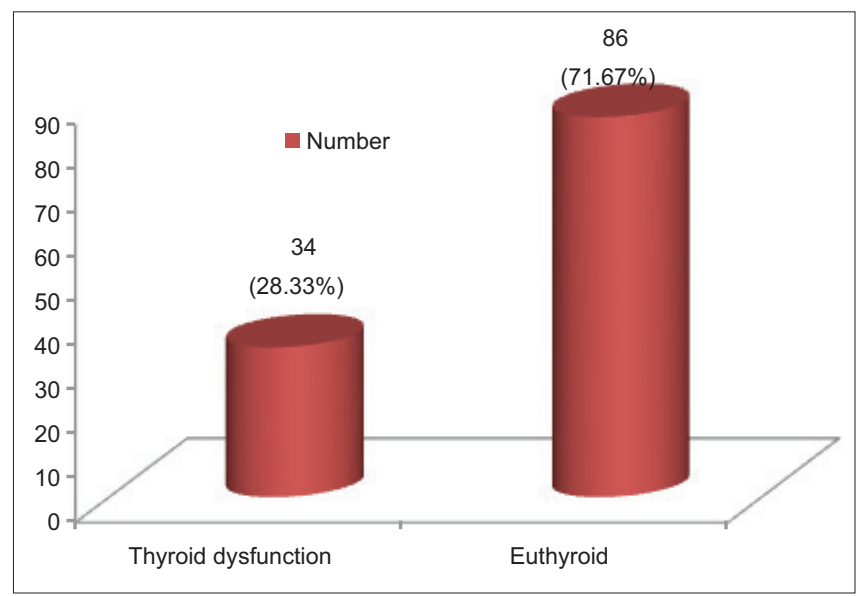

Figure 1: Prevalence of Thyroid dysfunction in Type 2 DM

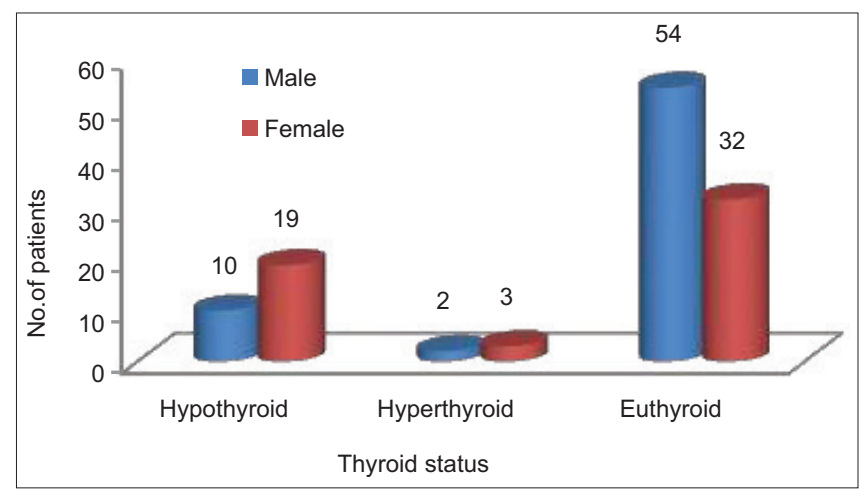

Figure 2: Gender distribution in type2 DM with thyroid dysfunction

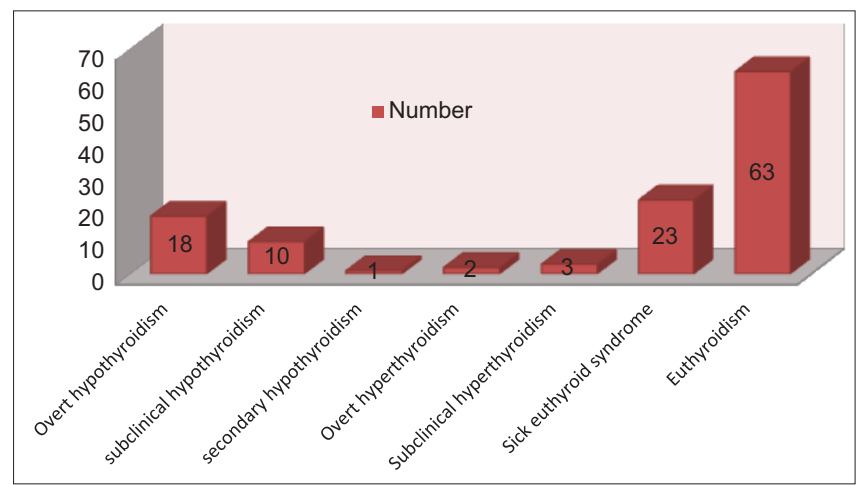

Figure 3: Categories of thyroid dysfunction in Type2 DM are an early sign of lymphocytic infiltration and they can be used as a predictor for the future development of hypothyroidism. In the 20-year follow-up study of the Wickham survey,the presence of TPO antibodies in females with a normal TSH level confined an annual risk of developing overt hypothyroidism of 2.1\%.21 This correlated with the antibody titre: if TPO antibodies were negative the risk was $4 \%$, if weakly positive $23 \%$, if moderately positively $33 \%$, and if strongly positive the risk increased to $53 \%$.

Regression analysis showed that TSH was positively correlated with anti TPO antibody $(r=0.4927)$ and the association was extremely significant $(\mathrm{p}<0.0001)$ as shown in (Table 1).

Pearson's correlation showed that, there was a significant negative correlation between TSH and fasting serum insulin, HOMA-IR and HOMA-B $(\mathrm{p}<0.05)$ as shown in (Table 2). It means high TSH was associated with low insulin resistance markers and low TSH was associated with higher insulin resistance and $\beta$-cell function.

The levels of T3, T4 were lower and TSH,anti TPO,antiTG \& ANA were significantly higher $(\mathrm{p}<0.05)$ in hypothyroid

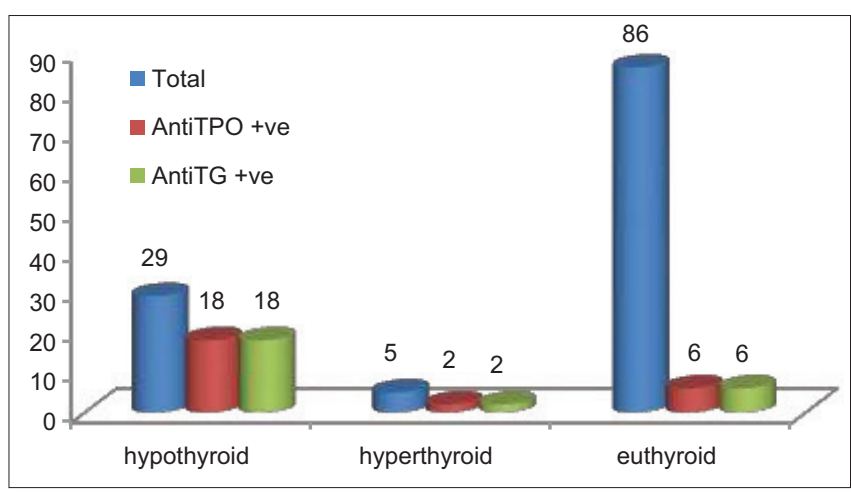

Figure 4: Presence Of Anti-TPO\& Anti-TG Antibodies In Type2 DM With Thyroid Dysfunction

\begin{tabular}{|c|c|c|c|}
\hline Parameters & TSH & Anti-TPO & $p$ value \\
\hline Mean & 6.29 & 55.33 & $<0.0001$ \\
\hline Median & 2.3 & 20 & Extremely \\
\hline SD & 16.016 & 110.37 & Significant \\
\hline
\end{tabular}

Table 2: Correlation between and fasting, Insulin, HOMA-IR and HOMA-B

\begin{tabular}{lccl}
\hline & r value & p value & Significance \\
\hline TSH vs fasting insulin & -0.29 & 0.0009 & Extremely significant \\
TSH vs HOMA-IR & -0.21 & 0.0179 & Significant \\
TSH vs HOMA-B & -0.2451 & 0.007 & Very significant \\
\hline
\end{tabular}


diabetic subjects than in euthyroid subjects. They had significantly lower values of fasting serum insulin, HOMAIR, HOMA-B than that of euthyroid as shown in (Table 3). Compared to euthyroid,hypothyroid diabetics were older in age.(p :0.0294). Neither hypothyroid nor hyperthyroid subjects showed significant difference in FBS,2hrPPBS,HbA1C,lipid profile

\section{DISCUSSION}

Prevalence of thyroid dysfunction in type 2 diabetes mellitus was $28.33 \%$ according to our study, which included overt hypothyroidism (15\%), subclinical hypothyroidism $(8.33 \%)$,secondary hypothyroidism( $0.83 \%)$, overt hyperthyroidism(1.67\%) and subclinical hyperthyroidism(2.5\%) as shown in (Figure 3). Recently a prevalence of $12.3 \%$ was reported among Greek diabetic patients by Papazafiropoulou et al $2010 .{ }^{11}$ In another study done by Gang Chen et al (2010), thyroid dysfunction was observed in $12.1 \%$ diabetic patients. Palma et al (2013) found that prevalence of thyroid dysfunction in type $2 \mathrm{DM}$ was $13.11 \%$. A retrospective study of thyroid dysfunction in type 2 diabetes mellitus revealed prevalence of $31.2 \%$ (Demitrost L et al). ${ }^{22}$

Bassyouni et al (2010) found a higher prevalence of thyroid dysfunction in older subjects with type 2 DM. Our study also revealed an increase in thyroid dysfunction with increasing age up to 69 year after which it decreased.

Anti TPO and anti TG antibodies were elevated in $62.07 \%$ cases of hypothyroidism, $40 \%$ cases of hyperthyroidism and

\begin{tabular}{|c|c|c|c|}
\hline Parameters & $\begin{array}{l}\text { Euthyroidism } \\
\qquad(n=86)\end{array}$ & $\begin{array}{l}\text { Hypothyroidism } \\
(n=29)\end{array}$ & $P$ value \\
\hline Age (years) & $60.96 \pm 14.47$ & $62.03 \pm 13.2$ & 0.0294 \\
\hline FBS & $222.59 \pm 108.6$ & $220.49 \pm 133.1$ & 0.448 \\
\hline $2 \mathrm{hr}$ PPBS & $280.18 \pm 104.33$ & $273.53 \pm 144.74$ & 0.4509 \\
\hline $\mathrm{HbA1c}$ & $8.57 \pm 1.38$ & $8.46 \pm 2.02$ & 0.4384 \\
\hline TC & $168.6 \pm 54.92$ & $179.65 \pm 56.79$ & 0.1552 \\
\hline TG & $166.13 \pm 113.08$ & $222.14 \pm 178.41$ & 0.0544 \\
\hline HDL & $40.78 \pm 11.04$ & $38.96 \pm 8.32$ & 0.3251 \\
\hline LDL & 10232.31 & $109.34 \pm 36.24$ & 0.0726 \\
\hline VLDL & $27.29 \pm 11.97$ & $25.1 \pm 10.22$ & 0.2236 \\
\hline TSH & $2.01 \pm 1.23$ & $20.03 \pm 28.87$ & $<0.0001$ \\
\hline Т3 & $0.83 \pm 0.49$ & $0.7 \pm 0.5$ & 0.0087 \\
\hline $\mathrm{T} 4$ & $8.36 \pm 22.16$ & 4.192 .29 & 0.0002 \\
\hline TPO Ab +ve & $36.34 \pm 90.6$ & $106.49 \pm 145.5$ & $<0.0001$ \\
\hline TG Ab +ve & $151.66 \pm 199.53$ & $384.41 \pm 260.85$ & $<0.0001$ \\
\hline ANA & $0.54 \pm 0.94$ & $1.47 \pm 2.05$ & $<0.0001$ \\
\hline Fasting insulin & $25.33 \pm 13.14$ & $17.16 \pm 14.16$ & 0.0044 \\
\hline HOMA-IR & $14.03 \pm 10.43$ & $12.18 \pm 18.52$ & 0.006 \\
\hline HOMA-B & $89.91 \pm 112.84$ & $57.45 \pm 77.87$ & 0.0043 \\
\hline
\end{tabular}

$6.9 \%$ euthyroid cases of type $2 \mathrm{DM}$ as shown in (Figure 4). Anti TPO and anti-TG antibodies were significantly raised in type $2 \mathrm{DM}$ patient with hypothroidism than that of euthyroid ( $p$ value $<0.0001)$.

Compared to euthyroid diabetics, hypothyroid cases had lower values of insulin resistance markers like fasting insulin, HOMA-IR and HOMA-B as shown in (Table 3). Hyperthyoid cases had higher values. It signifies that hyperthyroid diabetics have higher insulin resistance. Fasting insulin, HOMA-IR, HOMA-B showed negative correlation with TSH ( $\mathrm{p}$ value $<0.05)$.

The insulin levels were increased in this study partly because of increased glucose-stimulated insulin secretion (GSIS). Another important reason for the increase was insulin resistance which is common in hyperthyroidism. In hyperthyroidism, pancreatic $\beta$-cells secrete more insulin to compensate for the increased demand of insulin when plasma glucose increases and IR exists, which leads to an apparent increase in $\beta$-cell secretory function (a higher HOMA- $\beta$ ) in this study.

However in this study, we did not find the evidence of impaired $\beta$-cell function in hyperthyroidism which may be explained by compensatory hyperfunction of $\beta$-cell. As reported in a recent follow-up study, HOMA $\beta$-cell function increased between 3 and 4 years before diagnosis and then decreased until diagnosis of diabetes, which suggested that it would take several years before the impairment of $\beta$-cell function. As hyperthyroid subjects were diagnosed recently, so the course of thyroid disorder was not long enough to impair $\beta$-cell function.

In most other studies, the concentration of insulin in hypothyroidism is reported to be normal ${ }^{23}$ or decreased, ${ }^{24,25}$ which is similar to our result. In hypothyroidism, glucose homeostasis is affected due to reduced intestinal glucose absorption, hepatic gluconeogenesis and glycogenolysis and peripheral tissue glucose disposal.

Insulin sensitivity in hypothyroid patients has been found to be normal or decreased. Hypothyroidism, even in the subclinical stage, has been shown to be associated with insulin resistance. ${ }^{3,26}$ Our findings were different from these studies. A significant lower HOMA-IR (associated with a lower HOMA- $\beta$ ) was observed when we analyzed the participants with markedly elevated TSH levels suggesting that insulin sensitivity was increased in this group. Only two studies reported an increased sensitivity of glucose disposal to insulin in hypothyroidism, ${ }^{27}$ which was somewhat similar to this study.

In order to further understand the relationship between thyroid function, insulin resistance and insulin secretion 
of $\beta$-cells, a multivariate linear regression analysis with HOMA-IR or HOMA- $\beta$ as the outcome was used. We found a significant negative correlation between TSH and IR assessed by HOMA-IR index, which was contrary to other studies. ${ }^{28,29,30}$ In a trial with thyroid hormones in obesity, a positive correlation between IR parameters and serum TSH was significantly influenced by the BMI. ${ }^{30} \mathrm{In}$ fact, most of the IR parameters were related to obesity and dyslipidemia. Some studies also suggested that thyroid function might cause dyslipidemia through altered insulin sensitivity in healthy subjects ${ }^{31}$ and patients with type 2 diabetes mellitus. ${ }^{32}$ However, we found that HOMA-IR was diminished in the group with serum $\mathrm{TSH}>10 \mu \mathrm{U} /$ $\mathrm{ml}$, even though TG, LDL levels were significantly higher in this group suggesting that obesity and dyslipidemia in hypothyroidism were not associated with IR in this study.

There are some other mechanisms that may be responsible for these observations. Generally, there is a negative correlation between TSH and thyroid hormones. The higher serum TSH usually means the lower thyroid hormones via negative feedback. HOMA-IR decreased as TSH increased, which may be interpreted as a negative correlation between insulin sensitivity and thyroid hormones. It is well known that thyroid hormones have insulin antagonistic effects. As TSH increased, thyroid hormones decreased and insulin antagonistic effects weakened. It may be the main mechanism for these results. Besides, it is also possible that the course of thyroid disorder in this study was not long enough to impact the insulin sensitivity in hypothyroidism. On the other hand, the negative correlation between serum TSH and HOMA- $\beta$ in this study could be interpreted as an influence of the altered insulin sensitivity on the secretion of $\beta$-cells.

\section{CONCLUSION}

The prevalence of thyroid dysfunction in type 2 diabetes mellitus was $28.33 \%$ according to our study. The most common thyroid abnormality was overt hypothyroidism $(15 \%)$. Anti TPO and anti-TG antibodies were positive in $62.07 \%$ cases of hypothyroidism and $40 \%$ cases of hyperthyroidism. TSH showed significant positive correlation with anti TPO and anti-TG antibodies $(\mathrm{p}<0.0001)$. Our study demonstrated significant negative correlation between TSH and indices of insulin resistance and beta cell function (HOMA-IR, HOMA-B) [p<0.05].

\section{ABBREVIATIONS}

ANA-antinuclear antibody,DM-Diabetes mellitus, FPG-Fasting plasma glucose,GLUT: Glucose transporter, HbA1c-Glycosylated haemoglobin, HOMA-IR- homeostatic model assessment for insulin resistance, HOMA-B-homeostatic model assessment for beta cell function, RIA-radioimmuno assay, TH-Thyroid Hormone, TSH-Thyroid stimulating hormone, TPO-thyroid peroxidise, TG-thyroglobulin.

\section{REFERENCES}

1. Brenta G. Diabetes and thyroid disorders. The British Journal of Diabetes and Vascular Disease 2010; 10:172-177.

2. Chen HS, Wu TE, Jap TS, Lu RA, Wang ML, Chen RL, et al. Subclinical hypothyroidism is a risk factor for nephropathy and cardiovascular diseases in Type 2 diabetic patients. Diabetes Medicine 2007; 24(12):1336-1344.

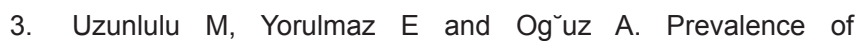
subclinical hypothyroidism in patients with metabolic syndrome. Endocrinology Journal 2007; 54:71-76.

4. IDF Diabetes Atlas 6th edition; 2014 update

5. Turnbridge WMJ and Evered DC. "The spectrum of thyroid disease in a community: The Wickham survey" Clinical Endocrinology 1977; 7(6): 481-493.

6. Junik R and Kozinski M. Thyroid ultrasound in Diabetic Patients without overt thyroid disease. Acta Radiologica 2006; 47(7): 687-691.

7. Park YS and Kim TW. Increased prevalence of autoimmune thyroid disease in patients with type I Diabetes. Korean Journal of Internal Medicine 2000; 15(3)202-210.

8. Menon PS and Vaidyanathan B. Autoimmune thyroid disease in Indian children with type 1 diabetes mellitus. Journal of paediatric endocrinology and metabolism 2001; 14(3):279-286.

9. Matejkova Behanova $M$ and Zamrazil V. Autoimmune thyroiditis in non obese subjects with initial diagnosis of type 2 diabetes mellitus. Journal of endocrinological investigations 2002;25(9) 779-784.

10. Papazafiropoulou A. Prevalence of thyroid dysfunction in Greek type 2 diabetic patients attending an outpatient clinic. Journal of clinical medicine research 2010;2(2): 75-78.

11. Swamy RM, Naveen Kumar, Srinivasa K, Manjunath GN, Prasad Byrav DS, Venkatesh G. Evaluation of hypothyroidism as a complication in Type II Diabetes Mellitus. Biomedical research 2012: 23(2) 170-172.

12. Raboudi N, Arem R, Jones RH, Chap Z, Pena J, Chou J, et al. Fasting and postabsorptive hepatic glucose and insulin metabolism in hyperthyroidism. Am J Physiol Endocrinol Metab 1989; 256: E159-E166.

13. Weinstein SP, O' Boyle E, Fisher M and Haber RS. Regulation of GLUT2 glucose transporter expression in liver by thyroid hormone: evidence for hormonal regulation of the hepatic glucose transport system. Endocrinology 1994; 135:649-654.

14. Viguerie N, Millet L, Avizou S, Vidal H, Larrouy D and Langin D. Regulation of human adipocyte gene expression by thyroid hormone. Journal of Clinical Endocrinology and Metabolism 2002; 87 630-634.

15. Clement $\mathrm{K}$, Viguerie $\mathrm{N}$, Diehn $\mathrm{M}$, Alizadeh $\mathrm{A}$, Barbe $\mathrm{P}$, Thalamas $\mathrm{C}$, et al. In Vivo Regulation of Human Skeletal Muscle Gene Expression by Thyroid Hormone. Genome Research 2002; 12:281-291.

16. Rochon $\mathrm{C}$ and Tauveron I. Response of glucose disposal to hyperinsulinemia in human hypothyroidism and hyperthyroidism. Clinical Science 2003; 104:7-15.

17. Handisurya A and Pacini G. Effects of thyroxin replacement 
therapy on glucose metabolism in subjects with subclinical and overt hypothyroidism. Clinical Endocrinology (OXFORD) 2008;69: 963-969.

18. Muller MJ, Paschen U and Seitz HJ. Thyroid hormone regulation of glucose homeostasis in the miniature pig. Endocrinology 1983; 112:2025-2031.

19. Okajima F and Ui M. Metabolism of glucose in hyper and hypothyroid rats in vivo. Glucose turnover values and futile cycle activities obtained with $14 \mathrm{C}$ and $3 \mathrm{H}$ labelled glucose. Biochemistry Journal 1979; 182:565-575.

20. McCulloch AJ, Nosadini R, Pernet A, Piniewska M, Cook DB, Clark F, et al. Glucose turnover and indices of recycling in thyrotoxicosis and primary thyroid failure. Clinical Science 1983; 64:41-47.

21. Prummel MF and Wiersinga WM. Thyroid peroxidase autoantibodies in euthyroid subjects. Best Practice and Research Clinical Endocrinology and Metabolism2005; 19(1):1-15.

22. Demitrost $L$ and Ranavir $S$. Thyroid dysfunction in type 2 diabetes mellitus: A retrospective study.Indian Journal of Endocrinology and Metabolism 2012; 16 (Suppl 2):s334-s335.

23. Renauld A and Sverdlik RC. Effects of hypothyroidism on serum immunoreactive insulin, free fatty acids and blood sugar in the dog as tested for oral glucose tolerance test: Corrective effects of thyroxine therapy. Hormone and Metabolic Research 1974;6:137-141.

24. Ahren B and Lundquist I. Glucose tolerance and insulin \& C-peptide responses after various insulin secretory stimuli in hyper and hypothyroid subjects before and after treatment. Diabetes Research 1985;2:95-103.
25. Katsilambros $\mathbf{N}$ and Ziegler R. Intravenous glucose tolerance and insulin secretion in the rat after thyroidectomy. Hormone and metabolic research 1972;4:377-379.

26. Dessein $\mathrm{PH}$ and Joffe BI. Subclinical hypothyroidism is associated with insulin resistance in rheumatoid arthritis. Thyroid 2004;14:443-446.

27. Jackson $I M$ and Prentice $C R$. The effect of hypothyroidism on glucose tolerance and insulin metabolism. Journal of Endocrinology 1970; 47:257-258.

28. Fernandez Real JM and Lopez Bermejo A. Thyroid function is intrinsically linked to insulin sensitivity and endothelium dependent vasodilation in healthy euthyroid subjects. Journal of Clinical Endocrinology \& Metabolism 2006;91:3337-3343.

29. Roos A and Bakker SJ. Thyroid function is associated with components of the metabolic syndrome in euthyroid subjects. Journal of Clinical Endocrinology \& Metabolism 2007;92: 491-496.

30. lacobellis $G$ and Ribaudo MC. Relationship of thyroid function with bodymass index, leptin, insulin sensitivity and adiponectin in euthyroid obese women. Clinical Endocrinology 2005;62; 487-491.

31. Bakker SJ and Maaten JC. The relationship between thyrotropin and LDL is modified by insulin sensitivity in healthy euthyroid subjects. Journal of Clinical Endocrinology \& Metabolism 2001;86:1206-1211.

32. Chubb SA and Davis WA. Interactions among thyroid function, insulin sensitivity and serum lipid concentrations; The Fremantle Diabetes study; Journal of Clinical Endocrinology \& Metabolism $2005 ; 90: 5317-5320$

\footnotetext{
Authors Contribution:

SS: Conception and design, acquisition of data. SKD: Analysis and interpretation of data. SSK: Drafting the article, Critical revision of the article. MMN: Critical revision of the article. PK: Conception and design, acquisition of data. UD: Critical revision of the article, final approval of the version to be published.
}

Source of Support: Nil, Conflict of Interest: None declared. 